\title{
Analysis of discrimination reversal learning in children
}

\author{
REESA M. VAUGHTER ${ }^{2}$, TEXAS TECHNOLOGICAL \\ COLLEGE, Lubbock, Texas
}

Children of two age groups were given discrimination reversal training. Variables were number of acquisition trials, type of prereversal training (PRT), and amount of PRT. On a given problem, $S$ received either criterion learning or overlearning on an object discrimination, and following either two, four, or eight trials on one of the four kinds of PRT conditions, six reversal trials. The four kinds of PRT conditions were Positive (POS), Negative (Neg), Mixed (Mix), and None (No). Mix PRT benefited $S$ more than Pos, Neg, or No PRT. Pos and Neg PRT benefited $S$ more than No PRT. Reversal performance following the Pos condition did not differ significantly from that following the Neg condition. Discrimination reversal performance was not affected by overlearning; however, discrimination reversal performance following eight PRT trials was better than that following two or four PRT trials. The findings were interpreted as lending support to a duoprocess theory of discrimination learning.

Studies have repeatedly shown (Harlow, 1959) that the performance of infrahuman primates given single-object training trials with a nonrewarded object, the Negative (Neg) Stimulus, will, in subsequent two-choice discrimination trials, exceed that of animals given comparable training trials with a rewarded object, the Positive (Pos) Stimulus. This phenomenon has become known as the Moss-Harlow effect (Moss \& Harlow, 1947), and the phenomenon lends support to a uniprocess, inhibition theory of discrimination learning. Employing the Moss-Harlow procedure (Moss \& Harlow, 1947), Cross \& Vaughter (1966) observed the MossHarlow effect in the discrimination performance of younger, but not older children; i.e., the younger children's performance was better following training with the Neg stimulus while the older children's performance was better following training with the Pos stimulus.

The Moss-Harlow effect has been observed in squirrel monkeys even when the single-object training trials were interposed between the acquisition and reversal phases of discrimination reversal (Cross \& Brown, 1965; Cross, Fickling, Carpenter, \& Brown, 1964). However, when Vaughter \& Cross (1965) employed the prereversal discrimination procedure with five-year-old children, the Moss-Harlow effect was not observed. These children showed the greatest reversal learning following either training with both the Pos and Neg stimuli or with the Pos stimulus alone. Training with the Neg stimulus alone was no more beneficial than a control situation involving no single-object, prereversal training trials.

The present study was designed to test for the occurrence of the Moss-Harlow effect in the performance of younger children when the prereversal discrimination procedure was used to test the effect. Both older and younger children were tested in order that an immediate comparison could be made; however, it was hypothesized that the older children's performance would replicate the findings of Vaughter \& Cross (1965). In addition, the study was designed to investigate the effects of the amount of single-object prereversal training (PRT) and the amount of overlearning in the discrimination training upon discrimination reversal performance. The nature of the relationships between these variables might be of significance to any theory proposing that discrimination reversal does not involve extinction of one habit and acquisition to a new one (Lovejoy, 1966).

Method. Thirty normal children, 15 below five years of age with a mean age of three years and four months, and 15 above five years of age with a mean age of five years and four months from the Lubbock Day School nurseries were employed as Ss.

A modified WGTA for children was utilized; the apparatus has been described in detail elsewhere (Olson, Cross, \& Vaughter, 1966). The essential features of the WGTA are a divided tabletop with a sliding three-well test tray and a one-way mirror assuring $\mathrm{E}$ a clear view of $\mathrm{S}$. The stimuli consisted of 48 multidimensional or "junk" objects mounted upon uniform bases and randomly paired to constitute 24 sets.
After meeting the child in the playroom of the nursery, E took $S$ to a testing room and seated him at the WGTA. After asking the $S$ if he liked candy, E pushed out the three-well test tray with a piece of candy in the center well and a plain adaptation block behind the baited food-well. The $E$ invited $S$ to eat the candy. On the next trial, the block partially obscured the candy, and on the third trial the block completely covered the candy. The $E$ then extended the test tray displaying the test objects.

The experimental design took the form of a split-plot analysis of variance in which age was a between-groups variable and initial problem length, kind of PRT, and amount of PRT were repeated measures. All 30 Ss received 24 problems which were factorial combinations of two initial problem lengths criterion learning and overlearning, four PRT conditions (None, Positive, Negative, Mixed), and three amounts of PRT (two, four, or eight trials). On a given problem, then, $S$ received either criterion learning or overlearning on an object discrimination and, following either two, four, or eight trials on one of the four kinds of PRT conditions, $\mathrm{S}$ was finally given six reversal trials.

Learning of the discrimination met criterion on a given problem when five out of six consecutive correct responses had been made. Overlearning consisted of 18 postcriterion trials.

For the Positive (Pos) prereversal condition, the $S$ received rewarded single-object training trials with the object that had initially been negative but which was to be positive during the six subsequent reversal trials; and, conversely, for the Negative (neg) condition $S$ received nonrewarded trials with the object which had initially been positive but was to be negative during reversal. For the Mixed (Mix) condition, the $\mathrm{S}$ received both Pos and Neg training trials, and the order of presentation was randomly determined for each S. For the None (No) condition, the reward contingencies were reversed for the two stimuli with no PRT provided.

Each $S$ received one problem per day. The order of the presentation of problems was randomized independently for each $S$. The position of the correct stimulus also randomized each trial within each problem for each $S$, with the restriction that the correct stimulus appeared as many times in the left as in the right position for any single problem. All PRT trials were given via the renter well.

For those variables where significant F-Ratios were found, all possible paired comparisons were made using the Tukey HSD Test. All response measures were subjected to arc sine transformation as recommended by Winer (1962)

Results. Analysis was performed on the number of correct choices made during the six reversal trials. The variables for which significant results were obtained were kind of PRT (F $=25.96$, df $=3 / 84, \mathrm{p}<.01)$ and amount of PRT $(F=3.51, \mathrm{df}=2 / 68, \mathrm{p}<$ .05 ). Subsequent tests showed that: (1) reversal performance following the Pos, Neg, or Mix training conditions was superior to that preceded by No PRT $(\mathrm{p}<.05)$; (2) reversal performance following Mix training was superior to that following either the Pos or Neg condition ( $p<.05)$; and $(3)$ reversal performance following the Pos condition did not differ significantly from that following the Neg training condition $(p>.05)$.

It was found that reversal performance was better following eight PRT trials $(p<.05)$, regardless of whether these were of the Pos, Neg, or Mix variety, than it was following four PRT trials. Four PRT trials resulted in better performance than did two PRT trials $(\mathrm{p}<.05)$.

Discussion. The discrimination reversal performance of the younger children of this study failed to demonstrate the Moss-Harlow effect. They, like the older children of this study and of a previous study (Vaughter \& Cross, 1965), demonstrated superior reversal performance following PRT with both the Pos and Neg stimuli. The present findings clearly support a duoprocess theory of discrimination learning which emphasizes the role of approach and avoidance, excitation and inhibition, respectively

The fact that PRT in either the Pos or Neg condition resulted in a significant reduction of errors in discrimination reversal suggests th t the two inferred processes are independent, i.e., either being a sufficient condition. one being a necessary condition for learning. Furthermore, since discrimination reversal performance following the Mix PRT was superior to that following either the Pos or Neg condition alone, it is hypothesized that the effectiveness of the two processes is the result of the summation of these processes; i.e., the two processes combine in an additive fashion to produce an even greater reduction of errors in discrimination reversal. 
Finally, although the number of discrimination reversals made was a function of the amount of PRT, overlearning had no effect upon correct choices in discrimination reversal, and this finding is not unique (Vaughter \& Cross, 1965). This finding is inconsistent with data presented by Cross \& Tyer (1966) in which the Overlearning Reversal Effect was observed in the performance of young children solving a position discrimination problem. Why the quantity of single-object PRT had an effect on correct choices in discrimination reversal while the quantity of two-object acquisition trials did not produce an effect is not obvious.

\section{REFERENCES}

CROSS, H. A., \& BROWN, L. T. Discrimination reversal learning in squirrel monkeys as a function of number of acquisition trials and prereversal experience. J. comp. physiol. Psychol., 1965, 59,429-431.

CROSS, H. A., FICKLING, R. N., CARPENTER, J. B., \& BROWN, L. T. Discrimination reversal performance in squirrel monkeys as a function of prereversal experience and overlearning. Psychon. Sci., 1964, 1, 353-354. CROSS, H. A., \& TYER, ZITA E. The overlearning reversal effect in preschool children as a function of age. Psychon. Sci., 1966, 6, 175-176.

CROSS, H. A., \& VAUGHTER, RESSA M. The Moss-Harlow effect in preschool children as a function of age. J. exp. child Psychol., 1966, 4, 280-284.
HARLOW, H. F. Learning set and error factor theory. In S. Koch (Ed.), Psychology: A study of a science. Vol. 2. New York: McGraw-Hill, 1959. Pp. $492-537$.

MOSS, EILEEN M., \& HARLOW, H. F. The role of reward in discrimination learning in monkeys. J. comp. physiol. Psychol., 1947, 40, 333-342.

OLSON, B. A., CROSS, H. A., \& VAUGHTER, REESA M. Apparatus note: A modified WGTA for children. Psychon. Sci., 1966, 5, 319.

VAUGHTER, REESA M., \& CROSS, H. A. Discrimination reversal performance in children as a function of prereversal experiemce and overlearning. Psychon. Sci., 1965, , 363-364.

VAUGHTER, REESA M. Analysis of discrimination reversal learning in children. Doctoral Dissertation, Texas Technological College, 1967.

WINER, B. J. Statistical principles in experimental design. New York: McGraw-Hill, 1962.

\section{NOTES}

1. This research was submitted to the faculty of Texas Technological College in partial fulfillment of the requirements for the Ph.D. degree. For an extensive review of the literature and detailed analysis of the data see Vaughter (1967).

2. Now at Department of Psychology, Northern Illinois University, DeKalb, Ill. 60115 . 